\title{
Stability Analysis of a Fractional-Order SEIR-KS Computer Virus-Spreading Model with Two Delays
}

\author{
Zhufeng Wang $\left(\mathbb{D},{ }^{1}\right.$ Xiaoqian Nie $\mathbb{D}^{2},{ }^{2}$ and Maoxin Liao $\mathbb{D}^{3}$ \\ ${ }^{1}$ Department of Foundation, Southwest Jiaotong University Hope College, Chengdu, Sichuan 610400, China \\ ${ }^{2}$ Department of Accounting, Southwest Jiaotong University Hope College, Chengdu, Sichuan 610400, China \\ ${ }^{3}$ School of Mathematics and Physics, University of South China, Hengyang, Hunan 421001, China
}

Correspondence should be addressed to Xiaoqian Nie; nxqthk@163.com

Received 12 August 2021; Accepted 8 October 2021; Published 19 October 2021

Academic Editor: Kolade M. Owolabi

Copyright (c) 2021 Zhufeng Wang et al. This is an open access article distributed under the Creative Commons Attribution License, which permits unrestricted use, distribution, and reproduction in any medium, provided the original work is properly cited.

In this paper, the stability and Hopf bifurcation of a fractional-order model of the Susceptible-Exposed-Infected-Kill Signals Recovered (SEIR-KS) computer virus with two delays are studied. The sufficient conditions for solving the stability and the occurrence of Hopf bifurcation of the system are established by using Laplace transform, stability theory, and Hopf bifurcation theorem of fractional-order differential systems. The research shows that time delays and fractional order $q$ have an important effect on the stability and the emergence of Hopf bifurcation of the fractional computer virus model. In addition, the validity of the theoretical analysis is verified by selecting appropriate system parameters for numerical simulation and the biological correlation of the equilibrium point is discussed. The results show that the bifurcation point of the model increases with the decrease in the model fractional order $q$. Under the same fractional order $q$, the effects of different types of delays on bifurcation points are obviously different.

\section{Introduction}

The spread of computer virus is the most common problem in network security. It spreads fast, spreads widely, and does serious harm. If it is not controlled in time, it will cause huge economic losses and seriously interfere with the normal production of the society. Therefore, it is very necessary to study the propagation mechanism of computer virus, and the research on virus propagation model has important significance both in theory and practice. In 1991, Kephart and White noted that there are many similarities between biological virus propagation and computer virus propagation [1]. It was the first time of introducing an epidemiological mathematical model into the study of computer virus transmission. Since then, many scholars have also used some models and analytical methods in biology to conduct indepth research on computer viruses [2-8].

The fractional-order model was first proposed in the study of basic mathematical theory, but it has not been widely used for a long time because of the complexity of its calculation [9]. With the development of computer technology, the complex calculation process can be completed by various software programs and the fractional model has reentered the field of view of researchers. Since fractional calculus can describe the evolutionary process with genetic attributes, its memory characteristics can not only describe the past state of the relevant system but also affect the present and future state of the system. In recent years, researchers in the fields of mathematics, physics, biology, and finance have found that some fractional models used to describe problems better reflect the real situation of the problem[10-16].

The proper "ecological memory" is an important characteristic of the biological population model; it refers to "the past state or experience affects the current or future response ability of the community" [17]. This characteristic coincides with that of fractional calculus. Many researchers have introduced fractional calculus into various models of biological mathematics and obtained a number of results with theoretical value and practical significance. For example, Djordjevic et al. used fractional-order ordinary differential 
equations to model a class of cell problems, and the consistency between the model and the actual data was more accurate than that of the integer-order model [18]. El-Saka studied the SIS epidemic model of fractional-order changing population and analyzed the stability of the equilibrium point and the conditions for the existence of the uniform steady-state solution [19]. Uçar et al. studied the stability of a fractional SAIDR model in the frame of the Atangana-Baleanu derivative [20]. The computer virus model based on epidemiology has a strong "memory" attribute because of its evolution process and control strategy and can be studied by using fractional calculus theory, so that new dynamic characteristics such as chaos can be obtained [21].

In this paper, a fractional reciprocal SEIR-KS computer virus model, which has double delay, is proposed on the basis of reference. Based on the conclusions summarized in [22], the relationship between Hopf bifurcation and time delay appearing at the positive equilibrium point of the model is theoretically analyzed. To verify the rationality of the theoretical analysis, the appropriate system parameters were selected for numerical simulation and the variation trend of the critical value of the time delay under the condition of fractional-order change was obtained through numerical simulation.

\section{Preliminaries and Model Description}

In this part, we will introduce the relevant theories of this study, which are about the Caputo fractional derivative and the theoretical research methods for the fractional-order computer virus-spreading model $[23,24]$.

First of all, we give the definition of the Caputo fractional-order derivative:

$$
D_{t}^{\alpha} f(t)=\frac{1}{\Gamma(u-\alpha)} \int_{0}^{t}(t-\tau)^{u-\alpha-1} f^{(n)}(\tau) \mathrm{d} \tau,
$$

where $u-1<\alpha<u, \mu \in N, 0 \leq \alpha \leq 1$, and $\Gamma(\cdot)$ is the Gamma function.

The definition of the Laplace transform of the Caputo fractional-order derivative is

$$
L\left\{D_{t}^{\alpha} f(t)\right\}=s^{\alpha} F(s)-\sum_{l=0}^{m-1} s^{\alpha-l-1} f^{(l)}(0) .
$$

If $f^{(l)}(0)=0$, where $l=0,1, \ldots, u-1$, we get $L\left\{D_{t}^{\alpha} f(t)\right\}=s^{\alpha} F(s)$.

Fangfang Yang and Zizhen Zhang studied the following integer-order time delay computer virus-spreading model [25]:

$$
\left\{\begin{array}{l}
\frac{\mathrm{d} S}{\mathrm{~d} t}=p b-\beta_{1} S(t) E(t)-\beta_{2} S(t) I(t)-\phi S(t) K(t)-\mu S(t)+\sigma R\left(t-\tau_{1}\right), \\
\frac{\mathrm{d} E}{\mathrm{~d} t}=r b+\beta_{1} S(t) E(t)+\beta_{2} S(t) I(t)-\alpha E\left(t-\tau_{2}\right)-\chi E(t)-\mu E(t), \\
\frac{\mathrm{d} I}{\mathrm{~d} t}=\alpha E\left(t-\tau_{2}\right)-\delta I(t) K(t)-\gamma I(t)-\varepsilon I(t)-\mu I(t), \\
\frac{\mathrm{d} K}{\mathrm{~d} t}=\delta I(t) K(t)+\gamma I(t)-\chi E(t)-\mu K(t), \\
\frac{\mathrm{d} R}{\mathrm{~d} t}=\phi S(t) K(t)+\varepsilon I(t)-\mu R(t)-\sigma R\left(t-\tau_{1}\right),
\end{array}\right.
$$

where $S(t)$ represent the number of susceptible computers, $E(t)$ represent the number of exposed computers, $I(t)$ represent the number of infected computers, $K(t)$ represent the number of kill signals computers, and $R(t)$ represent the number of recovered computers; $p, b, \beta_{1}, \beta_{2}, \phi, \mu, \sigma, \alpha, \chi, \delta, \gamma, \varepsilon, r \in(0,1)$ are the probability of interconversion between various nodes; $\tau_{1}$ is the temporary immunization time delay for the recovered computer to be converted into a susceptible computer; and $\tau_{2}$ is the latency from the exposed computer to the infected computer. They analyze the local stability and Hopf bifurcation of system (3) with time delay as the parameter and confirm the effectiveness of the conclusion. The direction of Hopf bifurcation and the stability of bifurcation periodic solution are proved.

Up to now, the Hopf bifurcation of the fractional computer virus model is poorly studied. To remedy this defect, we think that it is necessary to study the Hopf branch of the fractional computer virus. Inspired by the above analysis, on the basis of previous system (3), we establish the following fractional-order SEIR-KS computer virusspreading model with two delays: 


$$
\left\{\begin{array}{l}
D^{q} S(t)=p b-\beta_{1} S(t) E(t)-\beta_{2} S(t) I(t)-\phi S(t) K(t)-\mu S(t)+\sigma R\left(t-\tau_{1}\right), \\
D^{q} E(t)=r b+\beta_{1} S(t) E(t)+\beta_{2} S(t) I(t)-\alpha E\left(t-\tau_{2}\right)-\chi E(t)-\mu E(t), \\
D^{q} I(t)=\alpha E\left(t-\tau_{2}\right)-\delta I(t) K(t)-\gamma I(t)-\varepsilon I(t)-\mu I(t), \\
D^{q} K(t)=\delta I(t) K(t)+\gamma I(t)+\chi E(t)-\mu K(t), \\
D^{q} R(t)=\phi S(t) K(t)+\varepsilon I(t)-\mu R(t)-\sigma R\left(t-\tau_{1}\right),
\end{array}\right.
$$

where $q \in(0,1]$ is the fractional order. Obviously, system (4) degenerates into the model in [25] when $q=1$.

\section{The Stability and the Hopf Bifurcation Analysis}

In this section, some sufficient conditions are established, for the existence of Hopf bifurcation of system (4).

Make the five equations in system (4) be equal to zero. Next, we can obtain that

$\%\left\{\begin{array}{l}p b-\beta_{1} S(t) E(t)-\beta_{2} S(t) I(t)-\phi S(t) K(t)-\mu S(t)+\sigma R(t)=0, \\ r b+\beta_{1} S(t) E(t)+\beta_{2} S(t) I(t)-\alpha E(t)-\chi E(t)-\mu E(t)=0, \\ \alpha E(t)-\delta I(t) K(t)-\gamma I(t)-\varepsilon I(t)-\mu I(t)=0, \\ \delta I(t) K(t)+\gamma I(t)+\chi E(t)-\mu K(t)=0, \\ \phi S(t) K(t)+\varepsilon I(t)-\mu R(t)-\sigma R(t)=0 .\end{array}\right.$
Hence,

$$
\begin{aligned}
S^{*} & =\frac{\sigma R^{*}+p b}{\beta_{1} E^{*}+\beta_{2} I^{*}+\phi K^{*}+M}, \\
E^{*} & =\frac{r b}{\alpha+\chi+M-\beta_{1} E^{*}-\beta_{2} I^{*}}, \\
I^{*} & =\frac{\alpha E^{*}}{\delta K^{*}+\gamma+\varepsilon+M}, \\
K^{*} & =\frac{\gamma I^{*}+\chi E^{*}}{\mu-\delta I^{*}}, \\
R^{*} & =\frac{\varepsilon I^{*}+\phi S^{*} K^{*}}{\mu+\sigma} .
\end{aligned}
$$

From [25], if $R_{0}=\left(\beta_{1} b / \mu(\alpha+\chi+\mu)\right)>1, \quad\left(S^{*}, E^{*}, I^{*}\right.$, $\left.K^{*}, R^{*}\right)$ is a unique endemic equilibrium of system (4).

The linearized part of system (4) is

$$
\left\{\begin{array}{l}
D^{q} S(t)=a_{11} S(t)+a_{12} E(t)+a_{13} I(t)+a_{14} K(t)+m_{15} R\left(t-\tau_{1}\right), \\
D^{q} E(t)=a_{21} S(t)+a_{22} E(t)+n_{22} E\left(t-\tau_{2}\right)+a_{23} I(t), \\
D^{q} I(t)=n_{32} E\left(t-\tau_{2}\right)+a_{33} I(t)+a_{34} K(t), \\
D^{q} K(t)=a_{42} E(t)+a_{43} I(t)+a_{44} K(t), \\
D^{q} R(t)=a_{51} S(t)+a_{53} I(t)+a_{54} K(t)+a_{55} R(t)+m_{55} R\left(t-\tau_{1}\right),
\end{array}\right.
$$

where

$$
\begin{aligned}
& a_{11}=-\left(\beta_{1} E^{*}+\beta_{2} I^{*}+\phi K^{*}+\mu\right), \\
& a_{12}=-\beta_{1} S^{*}, \\
& a_{13}=-\beta_{2} S^{*}, \\
& a_{14}=\phi S^{*}, \\
& m_{15}=\sigma, \\
& a_{21}=\beta_{1} E^{*}+\beta_{2} I^{*}, \\
& a_{22}=\beta_{1} S^{*}-\chi-\mu, \\
& n_{22}=-\alpha, \\
& a_{23}=\beta_{2} S^{*}, \\
& n_{32}=\alpha,
\end{aligned}
$$

$$
\begin{aligned}
a_{33} & =-\left(\delta K^{*}+\gamma+\varepsilon+\mu\right), \\
a_{34} & =-\delta I^{*}, \\
a_{42} & =\chi, \\
a_{43} & =\delta K^{*}+\gamma, \\
a_{51} & =\phi K^{*}, \\
a_{53} & =\varepsilon, \\
a_{54} & =\phi S^{*}, \\
a_{55} & =-\mu, \\
m_{55} & =-\sigma, \\
a_{44} & =\delta I^{*}-\mu .
\end{aligned}
$$

We can get the characteristic equation of system (7): 


$$
\left|\begin{array}{ccccc}
\lambda^{q}-a_{11} & -a_{12} & -a_{13} & -a_{14} & -m_{15} e^{-\lambda \tau_{1}} \\
-a_{21} & \lambda^{q}-a_{22}-n_{22} e^{-\lambda \tau_{2}} & -a_{23} & 0 & 0 \\
0 & -n_{32} e^{-\lambda \tau_{2}} & \lambda^{q}-a_{33} & -a_{34} & 0 \\
0 & -a_{42} & -a_{43} & \lambda-a_{44} & 0 \\
-a_{51} & 0 & -a_{53} & -a_{54} & \lambda^{q}-a_{55}-m_{55} e^{-\lambda \tau_{1}}
\end{array}\right|=0
$$

Then, the following exponential equation can be obtained:

$$
\begin{aligned}
& \lambda^{5 q}+a_{4} \lambda^{4 q}+a_{3} \lambda^{3 q}+a_{2} \lambda^{2 q}+a_{1} \lambda^{q}++a_{0}+\left(b_{4} \lambda^{4 q}+b_{3} \lambda^{3 q}+b_{2} \lambda^{2 q}+b_{1} \lambda^{q}+b_{0}\right) e^{-\lambda \tau_{1}}+ \\
& \left(c_{4} \lambda^{4 q}+c_{3} \lambda^{3 q}+c_{2} \lambda^{2 q}+c_{1} \lambda^{q}+c_{0}\right) e^{-\lambda \tau_{1}}+\left(d_{3} \lambda^{3 q}+d_{2} \lambda^{2 q}+d_{1} \lambda^{q}+d_{0}\right) e^{-\lambda\left(\tau_{1}+\tau_{2}\right)}=0
\end{aligned}
$$

where

$$
\begin{aligned}
& a_{4}=-\left(a_{11}+a_{22}+a_{33}+a_{44}+a_{55}\right) \text {, } \\
& a_{3}=a_{11} a_{22}-a_{12} a_{21}+a_{11} a_{33}+a_{11} a_{33}+a_{11} a_{44}+a_{22} a_{33}+a_{11} a_{55}+ \\
& a_{22} a_{44}+a_{22} a_{55}+a_{33} a_{44}-a_{34} a_{43}+a_{33} a_{55}+a_{44} a_{55} \\
& a_{2}=-\left(a_{11} a_{22} a_{33}-a_{12} a_{21} a_{33}+a_{11} a_{22} a_{44}-a_{12} a_{21} a_{44}+a_{14} a_{21} a_{42}+a_{11} a_{22} a_{55}+a_{11} a_{33} a_{44}-a_{11} a_{34} a_{43}-a_{12} a_{21} a_{55}+\right. \\
& \left.a_{11} a_{33} a_{55}+a_{22} a_{33} a_{44}-a_{22} a_{34} a_{43}+a_{22} a_{34} a_{42}+a_{11} a_{44} a_{55}+a_{22} a_{33} a_{55}+a_{22} a_{44} a_{55}+a_{33} a_{44} a_{55}-a_{34} a_{43} a_{55}\right) \text {, } \\
& a_{1}=a_{11} a_{22} a_{33} a_{44}-a_{11} a_{22} a_{34} a_{43}+a_{11} a_{23} a_{34} a_{42}-a_{12} a_{21} a_{33} a_{44}+a_{12} a_{21} a_{34} a_{43}-a_{13} a_{21} a_{34} a_{42}+ \\
& a_{14} a_{21} a_{33} a_{42}+a_{11} a_{22} a_{33} a_{55}-a_{11} a_{21} a_{33} a_{55}+a_{11} a_{22} a_{44} a_{55}-a_{12} a_{21} a_{44} a_{55}+a_{14} a_{21} a_{42} a_{55}+ \\
& a_{11} a_{33} a_{44} a_{55}-a_{11} a_{34} a_{43} a_{55}+a_{22} a_{33} a_{44} a_{55}-a_{22} a_{34} a_{43} a_{55}+a_{23} a_{34} a_{42} a_{55} \text {, } \\
& a_{0}=-a_{11} a_{22} a_{33} a_{44} a_{55}+a_{11} a_{22} a_{34} a_{43} a_{55}-a_{11} a_{23} a_{34} a_{42} a_{55}+a_{12} a_{21} a_{33} a_{44} a_{55}- \\
& a_{12} a_{21} a_{34} a_{43} a_{55}+a_{13} a_{21} a_{34} a_{42} a_{55}-a_{14} a_{21} a_{33} a_{42} a_{55} \text {, } \\
& b_{4}=-m_{55} \text {, } \\
& b_{3}=a_{11} m_{55}+a_{51} m_{15}+a_{22} m_{55}+a_{33} m_{55}+a_{44} m_{55} \text {, } \\
& b_{2}=m_{55}\left(-a_{11} a_{22}+a_{12} a_{21}-a_{11} a_{33}-a_{11} a_{44}-a_{22} a_{33}-a_{22} a_{44}-a_{33} a_{44}+a_{34} a_{43}\right) \\
& +m_{15}\left(a_{22} a_{51}+a_{33} a_{51}+a_{44} a_{51}\right) \text {, } \\
& b_{1}=m_{55}\left(a_{11} a_{22} a_{33}-a_{12} a_{21} a_{33}+a_{11} a_{22} a_{44}-a_{12} a_{21} a_{44}+a_{14} a_{21} a_{42}+\right. \\
& \left.a_{11} a_{33} a_{44}-a_{11} a_{34} a_{43}+a_{22} a_{33} a_{44}-a_{22} a_{34} a_{43}+a_{23} a_{34} a_{42}\right) \text {, } \\
& b_{0}=m_{55}\left(-a_{11} a_{22} a_{33} a_{44}+a_{11} a_{22} a_{34} a_{43}-a_{11} a_{23} a_{34} a_{42}+a_{12} a_{21} a_{33} a_{44}-a_{12} a_{21} a_{34} a_{43}+a_{13} a_{21} a_{34} a_{42}-a_{14} a_{22} a_{33} a_{42}\right)+ \\
& m_{15}\left(a_{21} a_{33} a_{42} a_{54}-a_{21} a_{34} a_{42} a_{53}-a_{22} a_{33} a_{44} a_{51}-a_{22} a_{34} a_{43} a_{51}+a_{23} a_{34} a_{42} a_{51}\right) \text {, } \\
& c_{4}=-n_{22} \text {, } \\
& c_{3}=a_{11} n_{22}-a_{23} n_{32}+a_{33} n_{22}+a_{44} n_{22}+a_{55} n_{22} \text {, } \\
& c_{2}=-\left(\begin{array}{c}
-a_{11} a_{33} n_{22}+a_{13} a_{21} n_{32}+a_{11} a_{44} n_{22}+a_{11} a_{55} n_{22}-a_{23} a_{44} n_{32}+ \\
a_{33} a_{44} n_{22}-a_{34} a_{43} n_{22}-a_{23} a_{55} n_{32}+a_{33} a_{55} n_{22}+a_{44} a_{55} n_{22}
\end{array}\right) \text {, } \\
& c_{1}=n_{22}\left(a_{11} a_{33} a_{44}-a_{11} a_{34} a_{43}+a_{11} a_{33} a_{55}+a_{11} a_{44} a_{55}+a_{33} a_{44} a_{55}-a_{34} a_{43} a_{55}\right)+ \\
& n_{32}\left(-a_{11} a_{23} a_{44}+a_{13} a_{21} a_{44}-a_{14} a_{21} a_{43}-a_{11} a_{23} a_{55}+a_{13} a_{21} a_{55}-a_{23} a_{44} a_{55}\right), \\
& c_{0}=\left(a_{11} a_{23} a_{44} a_{55} a_{32}-a_{11} a_{33} a_{44} a_{55} a_{22}+a_{11} a_{34} a_{43} a_{55} a_{22}-a_{13} a_{21} a_{44} a_{55} a_{32}+a_{14} a_{21} a_{43} a_{55} a_{32}\right) \text {. }
\end{aligned}
$$


3.1. Case 1: $\tau_{1}=\tau_{2}=0$. When $\tau_{1}=\tau_{2}=0$, equation (10) becomes

$$
\lambda^{5}+B_{4} \lambda^{4}+B_{3} \lambda^{3}+B_{2} \lambda^{2}+B_{1} \lambda+B_{0}=0
$$

where

$$
\begin{aligned}
& B_{0}=a_{0}+b_{0}+c_{0}+d_{0}, \\
& B_{1}=a_{1}+b_{1}+c_{1}+d_{1}, \\
& B_{2}=a_{2}+b_{2}+c_{2}+d_{2}, \\
& B_{3}=a_{3}+b_{3}+c_{3}+d_{3}, \\
& B_{4}=a_{4}+b_{4}+c_{4}+d_{4} .
\end{aligned}
$$

By the Routh-Hurwitz criterion, a set of necessary and sufficient conditions for all roots of equation (14) to have a negative real part is given in the following form:

$$
\begin{aligned}
& D_{1}=B_{4}>0, \\
& D_{2}=\left|\begin{array}{cc}
B_{4} & B_{2} \\
1 & B_{3}
\end{array}\right|>0, \\
& D_{3}=\left|\begin{array}{ccc}
B_{4} & B_{2} & B_{0} \\
1 & B_{3} & B_{1} \\
0 & B_{4} & B_{2}
\end{array}\right|>0, \\
& D_{4}=\left|\begin{array}{cccc}
B_{4} & B_{2} & B_{1} & 0 \\
1 & B_{3} & B_{2} & 0 \\
0 & B_{4} & B_{2} & B_{0} \\
0 & 1 & B_{3} & B_{1}
\end{array}\right|>0,
\end{aligned}
$$

$$
D_{5}=\left|\begin{array}{ccccc}
B_{4} & B_{2} & B_{1} & 0 & 0 \\
1 & B_{3} & B_{2} & 0 & 0 \\
0 & B_{4} & B_{2} & B_{0} & 0 \\
0 & 1 & B_{3} & B_{1} & 0 \\
0 & 0 & B_{4} & B_{2} & B_{0}
\end{array}\right|>0
$$

Lemma 1. If $\tau_{1}=\tau_{2}=0, R_{0}>1$, and (16)-(20) are satisfied, the endemic equilibrium of system (4) is asymptotically stable.

3.2. Case 2: $\tau_{1}>0, \tau_{2}=0$. When $\tau_{1}>0$ and $\tau_{2}=0$, then we can obtain the following equation:

$$
\begin{aligned}
& \lambda^{5 q}+C_{4} \lambda^{4 q}+C_{3} \lambda^{3 q}+C_{2} \lambda^{2 q}+C_{1} \lambda^{q}+C_{0}+ \\
& \left(E_{4} \lambda^{4 q}+E_{3} \lambda^{3 q}+E_{2} \lambda^{2 q}+E_{1} \lambda^{q}+E_{0}\right) e^{-\lambda \tau_{1}}=0,
\end{aligned}
$$

where $C_{4}=a_{4}+c_{4}, C_{3}=a_{3}+c_{3}, C_{2}=a_{2}+c_{2}, C_{1}=a_{1}+c_{1}$, $C_{0}=a_{0}+c_{0}, \quad E_{4}=b_{4}, \quad E_{3}=b_{3}+d_{3}, \quad E_{2}=b_{2}+d_{2}$, $E_{1}=b_{1}+d_{1}$, and $E_{0}=b_{0}+d_{0}$.

Let $\lambda=\dot{i} \omega$; we substitute this into (21), and separate the real part from imaginary part:

$$
\left\{\begin{array}{l}
M_{11} \cos \left(\omega \tau_{1}\right)+M_{12} \sin \left(\omega \tau_{1}\right)=M_{13}, \\
M_{12} \cos \left(\omega \tau_{1}\right)-M_{11} \sin \left(\omega \tau_{1}\right)=M_{14}
\end{array}\right.
$$

where

$$
\begin{aligned}
& M_{11}=E_{4} \omega^{4 q} \cos (2 q \pi)+E_{3} \omega^{3 q} \cos \left(\frac{3 q \pi}{2}\right)+E_{2} \omega^{2 q} \cos (q \pi)+E_{1} \omega^{q} \cos \left(\frac{q \pi}{2}\right)+E_{0}, \\
& M_{12}=E_{4} \omega^{4 q} \sin (2 q \pi)+E_{3} \omega^{3 q} \sin \left(\frac{3 q \pi}{2}\right)+E_{2} \omega^{2 q} \sin (q \pi)+E_{1} \omega^{q} \sin \left(\frac{q \pi}{2}\right), \\
& M_{13}=\omega^{5 q} \cos \left(\frac{5 q \pi}{2}\right)+C_{4} \omega^{4 q} \cos (2 q \pi)+C_{3} \omega^{3 q} \cos \left(\frac{3 q \pi}{2}\right)+C_{2} \omega^{2 q} \cos (q \pi)+C_{1} \omega^{q} \cos \left(\frac{q \pi}{2}\right)+C_{0}, \\
& M_{14}=\omega^{5 q} \sin \left(\frac{5 q \pi}{2}\right)+C_{4} \omega^{4 q} \sin (2 q \pi)+C_{3} \omega^{3 q} \sin \left(\frac{3 q \pi}{2}\right)+C_{2} \omega^{2 q} \sin (q \pi)+C_{1} \omega^{q} \sin \left(\frac{q \pi}{2}\right) .
\end{aligned}
$$

From (22), it is easy to obtain

$$
\begin{aligned}
\cos \left(\omega \tau_{1}\right) & =\frac{M_{13} M_{11}+M_{14} M_{12}}{M_{11}^{2}+M_{12}^{2}}, \\
M_{11}^{2}+M_{12}^{2} & =M_{13}^{2}+M_{14}^{2} .
\end{aligned}
$$

By (25), we have

$$
\begin{aligned}
\omega^{10 q} & +f_{9} \omega^{9 q}+f_{8} \omega^{8 q}+f_{7} \omega^{7 q}+f_{6} \omega^{6 q}+f_{5} \omega^{5 q}+f_{4} \omega^{4 q} \\
& +f_{3} \omega^{3 q}+f_{2} \omega^{2 q}+f_{1} \omega^{q}+f_{0}=0,
\end{aligned}
$$

where 


$$
\begin{aligned}
& f_{0}=C_{0}^{2}-E_{0}^{2} \\
& f_{1}=2 C_{0} C_{1} \cos \left(\frac{q \pi}{2}\right)-2 E_{0} E_{1} \cos \left(\frac{q \pi}{2}\right) \\
& f_{2}=C_{1}^{2}-E_{1}^{2}+2 C_{0} C_{1} \cos (q \pi)-2 E_{0} E_{2} \cos (q \pi), \\
& f_{3}=2 C_{0} C_{3} \cos \left(\frac{3 q \pi}{2}\right)-2 E_{0} E_{3} \cos \left(\frac{3 q \pi}{2}\right)+2 C_{1} C_{2} \sin (q \pi) \sin \left(\frac{q \pi}{2}\right)- \\
& 2 E_{1} E_{2} \sin (q \pi) \sin \left(\frac{q \pi}{2}\right)+2 C_{1} C_{2} \cos (q \pi) \cos \left(\frac{q \pi}{2}\right)-2 E_{1} E_{2} \cos (q \pi) \cos \left(\frac{q \pi}{2}\right), \\
& f_{4}=C_{2}^{2}-E_{2}^{2}+2 C_{0} C_{4} \cos (2 q \pi)-2 E_{0} E_{4} \cos (2 q \pi)+2 C_{1} C_{3} \sin \left(\frac{q \pi}{2}\right) \sin \left(\frac{3 q \pi}{2}\right)- \\
& 2 E_{1} E_{3} \sin \left(\frac{q \pi}{2}\right) \sin \left(\frac{3 q \pi}{2}\right)+2 C_{1} C_{3} \cos \left(\frac{q \pi}{2}\right) \cos \left(\frac{3 q \pi}{2}\right)-2 E_{1} E_{3} \cos \left(\frac{q \pi}{2}\right) \cos \left(\frac{3 q \pi}{2}\right), \\
& f_{5}=2 C_{0} \cos \left(\frac{5 q \pi}{2}\right)+2 C_{1} C_{4} \sin (2 q \pi) \sin \left(\frac{q \pi}{2}\right)+2 C_{2} C_{3} \sin (q \pi) \sin \left(\frac{3 q \pi}{2}\right)-2 E_{1} E_{4} \sin (2 q \pi) \\
& \sin \left(\frac{q \pi}{2}\right)-2 E_{2} E_{3} \sin (q \pi) \sin \left(\frac{3 q \pi}{2}\right)+2 C_{1} C_{4} \cos (2 q \pi) \cos \left(\frac{q \pi}{2}\right) \\
& +2 C_{2} C_{3} \cos (q \pi) \cos \left(\frac{3 q \pi}{2}\right)-2 E_{1} E_{4} \cos (2 q \pi) \cos \left(\frac{q \pi}{2}\right)-2 E_{2} E_{3} \cos (q \pi) \sin \left(\frac{3 q \pi}{2}\right), \\
& f_{6}=C_{3}^{2}-E_{3}^{2}+2 C_{1} \cos \left(\frac{q \pi}{2}\right) \cos \left(\frac{5 q \pi}{2}\right)+2 C_{1} \sin \left(\frac{q \pi}{2}\right) \sin \left(\frac{5 q \pi}{2}\right)+2 C_{2} C_{4} \sin (q \pi) \sin (2 q \pi) \\
& -2 E_{2} E_{4} \sin (q \pi) \sin (2 q \pi)+2 C_{2} C_{4} \cos (q \pi) \cos (2 q \pi)-2 E_{2} E_{4} \cos (q \pi) \cos (2 q \pi), \\
& f_{7}=2 C_{2} \cos (q \pi) \cos \left(\frac{5 q \pi}{2}\right)+2 C_{2} \sin (q \pi) \sin \left(\frac{5 q \pi}{2}\right)+2 C_{3} C_{4} \sin (2 q \pi) \sin \left(\frac{3 q \pi}{2}\right)- \\
& 2 E_{3} E_{4} \sin (2 q \pi) \sin \left(\frac{3 q \pi}{2}\right)+2 C_{3} C_{4} \cos (2 q \pi) \cos \left(\frac{3 q \pi}{2}\right)-2 E_{3} E_{4} \cos (2 q \pi) \cos \left(\frac{3 q \pi}{2}\right) \text {, } \\
& f_{8}=C_{4}^{2}-E_{4}^{2}-2 C_{3} \cos \left(\frac{3 q \pi}{2}\right) \cos \left(\frac{5 q \pi}{2}\right)+2 C_{3} \sin \left(\frac{3 q \pi}{2}\right) \sin \left(\frac{5 q \pi}{2}\right), \\
& f_{9}=2 C_{4} \cos (2 q \pi) \cos \left(\frac{5 q \pi}{2}\right)+2 C_{4} \sin (2 q \pi) \sin \left(\frac{5 q \pi}{2}\right) \text {. }
\end{aligned}
$$

Let $\quad h_{1}(\omega)=\omega^{10 q}+f_{9} \omega^{9 q}+f_{8} \omega^{8 q}+f_{7} \omega^{7 q}+f_{6} \omega^{6 q}+$ $f_{5} \omega^{5 q}+f_{4} \omega^{4 q}+f_{3} \omega^{3 q}+f_{2} \omega^{2 q}+f_{1} \omega^{q}+f_{0} ; \quad$ obviously, $\lim _{\omega \longrightarrow+\infty} h_{1}(\omega)=+\infty$. When $f_{0}=C_{0}^{2}-E_{0}^{2}<0$, namely, $\left(a_{0}-C_{0}\right)^{2}-\left(b_{0}-d_{0}\right)^{2}<0$, then (26) has at least one positive real root.

Lemma 2. When $\left(a_{0}-C_{0}\right)^{2}-\left(b_{0}-d_{0}\right)^{2}<0$, (26) has at least one positive real root.

By (24), we have

$$
\tau_{10}=\frac{1}{\omega_{10}} \arccos \frac{M_{13} M_{11}+M_{14} M_{12}}{M_{11}^{2}+M_{12}^{2}},
$$

where $\pm i \omega_{10}$ is a pair of purely imaginary roots of (21) with $\tau_{10}$. Also,

$$
\text { (H1) }\left.\operatorname{Re}\left(\frac{d \lambda}{d \tau_{1}}\right)^{-1}\right|_{\tau_{1}=\tau_{10}} \neq 0 .
$$
obtain

Taking the derivative of $\lambda$ with respect to $\tau_{1}$ in (21), we can

$$
\frac{\mathrm{d} \lambda}{d_{\tau_{1}}}=\frac{\lambda V_{13}}{V_{11}+V_{12}-\tau_{1} V_{13}}
$$

where 


$$
\begin{aligned}
V_{11} & =5 q \lambda^{5 q-1}+4 q C_{4} \lambda^{4 q-1}+3 q C_{3} \lambda^{3 q-1}+2 q C_{2} \lambda^{2 q-1}+q C_{1} \lambda^{q-1}, \\
V_{12} & =\left(4 q E_{4} \lambda^{4 q-1}+3 q E_{3} \lambda^{3 q-1}+2 q E_{2} \lambda^{2 q-1}+q E_{1} \lambda^{q-1}\right) e^{-\lambda \tau_{1}}, \\
V_{13} & =\left(E_{4} \lambda^{4 q}+E_{3} \lambda^{3 q}+E_{2} \lambda^{2 q}+E_{1} \lambda^{q}+E_{0}\right)^{-\lambda \tau_{1}}, \\
\left(\frac{d \lambda}{d_{\tau_{1}}}\right)^{-1}= & \frac{V_{11}+V_{12}}{\lambda V_{13}}=-\frac{\tau_{1}}{\lambda}, \\
\left(\frac{d \lambda}{d_{\tau_{1}}}\right)^{-1}= & \frac{W_{11}+W_{12} \cos \left(\omega \tau_{1}\right)+W_{13} \sin \left(\omega \tau_{1}\right)+i\left(-W_{13} \cos \left(\omega \tau_{1}\right)-W_{11} \sin \left(\omega \tau_{1}\right)+W_{14}\right)}{W_{15} \cos \left(\omega \tau_{1}\right)+W_{16} \sin \left(\omega \tau_{1}\right)+i\left(-W_{16} \cos \left(\omega \tau_{1}\right)-W_{15} \sin \left(\omega \tau_{1}\right)\right)}-\frac{\tau}{i W},
\end{aligned}
$$

where

$$
\begin{aligned}
W_{11} & =5 q \omega^{5 q-1} \sin \left(\frac{5 q \pi}{2}\right)+4 q C_{4} \omega^{5 q-1} \sin (2 q \pi)+3 q C_{3} \omega^{3 q-1} \sin \left(\frac{3 q \pi}{2}\right)+2 q C_{2} \omega^{2 q-1} \sin (q \pi)+q C_{1} \omega^{q-1} \sin \left(\frac{q \pi}{2}\right), \\
W_{12} & =4 q E_{4} \omega^{4 q-1} \sin (2 q \pi)+3 q E_{3} \omega^{3 q-1} \sin \left(\frac{3 q \pi}{2}\right)+2 q E_{2} \omega^{2 q-1} \sin (q \pi)+q E_{1} \omega^{q-1} \sin \left(\frac{q \pi}{2}\right), \\
W_{13} & =4 q E_{4} \omega^{4 q-1} \cos (2 q \pi)+2 q E_{3} \omega^{3 q-1} \cos \left(\frac{3 q \pi}{2}\right)+2 q E_{2} \omega^{2 q-1} \cos (q \pi)+q E_{1} \omega^{q-1} \cos \left(\frac{q \pi}{2}\right), \\
W_{14} & =-\left(5 q \omega^{5 q-1} \cos \left(\frac{5 q \pi}{2}\right)+4 q C_{4} \omega^{4 q-1} \cos (2 q \pi)+3 q C_{3} \omega^{3 q-1} \cos \left(\frac{3 q \pi}{2}\right)+2 q C_{2} \omega^{2 q-1} \cos (q \pi)+q C_{1} \omega^{q} \cos \left(\frac{q \pi}{2}\right)\right), \\
W_{15} & =-\omega\left(E_{4} \omega^{4 q} \sin (2 q \pi)+E_{3} \omega^{3 q} \sin \left(\frac{3 q \pi}{2}\right)+E_{2} \omega^{2 q} \sin (q \pi)+E_{1} \omega^{q} \sin \left(\frac{q \pi}{2}\right)\right), \\
W_{16} & =\omega\left(E_{4} \omega^{4 q} \cos (2 q \pi)+E_{3} \omega^{3 q} \cos \left(\frac{3 q \pi}{2}\right)+E_{2} \omega^{2 q} \cos (q \pi)+E_{1} \omega^{q} \cos \left(\frac{q \pi}{2}\right)+E_{0}\right), \\
R_{e}\left(\frac{\mathrm{d} \lambda}{d_{\tau_{1}}}\right)^{-1} & =\frac{X_{11} X_{13}+X_{12} X_{14}}{X_{13}^{2}+X_{14}^{2}},
\end{aligned}
$$

where

$$
\begin{aligned}
& X_{11}=W_{11}+W_{12} \cos \left(\omega \tau_{1}\right)+W_{13} \sin \left(\omega \tau_{1}\right), \\
& X_{12}=-W_{13} \cos \left(\omega \tau_{1}\right)-W_{11} \sin \left(\omega \tau_{1}\right)+W_{14} \\
& X_{13}=W_{15} \cos \left(\omega \tau_{1}\right)+W_{16} \sin \left(\omega \tau_{1}\right) \\
& X_{14}=-W_{16} \cos \left(\omega \tau_{1}\right)-W_{15} \sin \left(\omega \tau_{1}\right) .
\end{aligned}
$$

Noticing that $\operatorname{sign}\left[\left.\operatorname{Re}\left(d \lambda / d \tau_{1}\right)\right|_{\tau_{1}=\tau_{10}}\right]=\operatorname{sign}[\operatorname{Re}(d \lambda /$ $\left.\left.d \tau_{1}\right)\left.^{-1}\right|_{\tau_{1}=\tau_{10}}\right]$, we can obtain $\left.\operatorname{Re}\left(d \lambda / d \tau_{1}\right)\right|_{\tau_{1}=\tau_{10}} \neq 0$.

Theorem 1. Suppose that $R_{0}>1$, (16)-(20), (H1), and $\left(a_{0}-C_{0}\right)^{2}-\left(b_{0}-d_{0}\right)^{2}<0$ hold; then, the following results hold:

(i) When $\tau_{1} \in\left[0, \tau_{10}\right)$, the endemic equilibrium $\left(S^{*}, E^{*}, I^{*}, K^{*}, R^{*}\right)$ is asymptotically stable (ii) When $\tau_{1}=\tau_{10}$, system (4) experiences a Hopf bifurcation at the endemic equilibrium. In other words, system (4) has a bifurcation of periodic solution bifurcating near $\tau_{1}=\tau_{10}$.

3.3. Case 3: $\tau_{1}=0, \tau_{1}<0$. When $\tau_{1}=0, \tau_{1}<0$, equation (10) becomes

$$
\begin{aligned}
& \lambda^{5 q}+F_{4} \lambda^{4 q}+F_{3} \lambda^{3 q}+F_{2} \lambda^{2 q}+F_{1} \lambda^{q}+F_{0}+\left(G_{4} \lambda^{4 q}+G_{3} \lambda^{3 q}\right. \\
& \left.+G_{2} \lambda^{2 q}+G_{1} \lambda^{q}+G_{0}\right) e^{-\lambda \tau_{1}}=0 .
\end{aligned}
$$

where $\quad F_{0}=a_{0}+b_{0}, \quad F_{1}=a_{1}+b_{1}, \quad F_{2}=a_{2}+b_{2}$, $F_{3}=a_{3}+b_{3}, \quad F_{4}=a_{4}+b_{4}, \quad G_{0}=c_{0}+d_{0}, \quad G_{1}=c_{1}+d_{1}$, $G_{2}=c_{2}+d_{2}, G_{3}=c_{3}+d_{3}$, and $G_{4}=c_{4}$.

Since (19) and (25) are the same equation, we can draw the conclusion directly: 


$$
\text { (H2) }\left.\operatorname{Re}\left(\frac{\mathrm{d} \lambda}{\mathrm{d} \tau_{2}}\right)^{-1}\right|_{\tau_{2}=\tau_{20}} \neq 0 \text {. }
$$

Theorem 2. Suppose that $R_{0}>1$, (16)-(20), (H2), and $\left(a_{0}+b_{0}\right)^{2}-\left(c_{0}+d_{0}\right)^{2}<0$ hold; then, the following results hold:

(i) When $\tau_{2} \in\left[0, \tau_{20}\right)$, the endemic equilibrium $\left(S^{*}, E^{*}, I^{*}, K^{*}, R^{*}\right)$ is asymptotically stable.

(ii) When $\tau_{2}=\tau_{20}$, system (4) experiences a Hopf bifurcation at the endemic equilibrium. In other words, system (4) has a bifurcation of periodic solution bifurcating near $\tau_{2}=\tau_{20}$.
3.4. Case 4: $\tau_{1}=\tau_{2}=\tau_{*}>0$. When $\tau_{1}=\tau_{2}=\tau_{*}>0$, we can easily obtain the following equation:

$$
\begin{aligned}
& \left(\lambda^{5 q}+a_{4} \lambda^{4 q}+a_{3} \lambda^{3 q}+a_{2} \lambda^{2 q}+a_{1} \lambda^{q}+a_{0}\right) e^{\lambda \tau_{*}}+ \\
& \left(J_{4} \lambda^{4 q}+J_{3} \lambda^{3 q}+J_{2} \lambda^{2 q}+J_{1} \lambda^{q}+J_{0}\right) \\
& +\left(d_{3} \lambda^{3 q}+d_{2} \lambda^{2 q}+d_{1} \lambda^{q}+d_{0}\right) e^{-\lambda \tau_{*}}=0,
\end{aligned}
$$

where $J_{0}=b_{0}+c_{0}, J_{1}=b_{1}+c_{1}, J_{2}=b_{2}+c_{2}, J_{3}=b_{3}+c_{3}$, and $J_{4}=b_{4}+c_{4}$.

Supposing $\lambda=\dot{i} \omega$, next we can obtain equation (38) by separating the real part from imaginary part:

$$
\left\{\begin{array}{l}
M_{31} \cos \left(\omega \tau_{*}\right)+M_{32} \sin \left(\omega \tau_{*}\right)=M_{33}, \\
M_{34} \cos \left(\omega \tau_{*}\right)-M_{35} \sin \left(\omega \tau_{*}\right)=M_{36},
\end{array}\right.
$$

where

$$
\begin{aligned}
M_{31}= & \omega^{5 q} \cos \left(\frac{5 q \pi}{2}\right)+a_{4} \omega^{4 q} \cos (2 q \pi)+a_{3} \omega^{3 q} \cos \left(\frac{3 q \pi}{2}\right)+a_{2} \omega^{2 q} \cos (q \pi)+ \\
& a_{1} \omega^{q} \cos \left(\frac{q \pi}{2}\right)+a_{0}+d_{3} \omega^{3 q} \cos \left(\frac{3 q \pi}{2}\right)+d_{2} \omega^{2 q} \cos (q \pi)+d_{1} \omega^{q} \cos \left(\frac{q \pi}{2}\right)+d_{0}, \\
M_{32}= & -\left(\omega^{5 q} \sin \left(\frac{5 q \pi}{2}\right)+a_{4} \omega^{4 q} \sin (2 q \pi)+a_{3} \omega^{3 q} \sin \left(\frac{3 q \pi}{2}\right)+a_{2} \omega^{2 q} \sin (q \pi)+a_{1} \omega^{q} \sin \left(\frac{q \pi}{2}\right)\right), \\
M_{33}= & J_{4} \omega^{4 q} \cos (2 q \pi)+J_{3} \omega^{3 q} \cos \left(\frac{3 q \pi}{2}\right)+J_{2} \omega^{2 q} \cos (q \pi)+J_{1} \omega^{q} \cos \left(\frac{q \pi}{2}\right)+J_{0}, \\
M_{34}= & \omega^{5 q} \sin \left(\frac{5 q \pi}{2}\right)+a_{4} \omega^{4 q} \sin (2 q \pi)+a_{3} \omega^{3 q} \sin \left(\frac{3 q \pi}{2}\right)+a_{2} \omega^{2 q} \sin (q \pi)+a_{1} \omega^{q} \sin \left(\frac{q \pi}{2}\right)+d_{3} \omega^{3 q} \sin \left(\frac{3 q \pi}{2}\right) \\
& +d a_{2} \omega^{2 q} \sin (q \pi)+d_{1} \omega^{q} \sin \left(\frac{q \pi}{2}\right), \\
& -\left(d_{3} \omega^{3 q} \cos \left(\frac{3 q \pi}{2}\right)+d_{2} \omega^{2 q} \cos (q \pi)+d_{1} \omega^{q} \cos \left(\frac{q \pi}{2}\right)+d_{0}\right), \\
M_{36}= & J_{4} \omega^{4 q} \sin (2 q \pi)+J_{3} \omega^{3 q} \sin \left(\frac{3 q \pi}{2}\right)+J_{2} \omega^{2 q} \sin (q \pi)+J_{1} \omega^{q} \sin \left(\frac{q \pi}{2}\right) . \\
M_{35}= & \omega^{5 q} \cos \left(\frac{5 q \pi}{2}\right)+a_{4} \omega^{4 q} \cos (2 q \pi)+a_{3} \omega^{3 q} \cos \left(\frac{3 q \pi}{2}\right)+a_{2} \omega^{2 q} \cos (q \pi)+a_{1} \omega^{q} \cos \left(\frac{q \pi}{2}\right)+a_{0}
\end{aligned}
$$

Suppose that (38) has roots, the expression of $\sin (\omega \tau)$ and $\cos (\omega \tau)$ can be obtained as follows: 


$$
\begin{aligned}
& \cos \left(\omega \tau_{*}\right)=\frac{M_{33} M_{35}-M_{32} M_{36}}{M_{31} M_{35}-M_{32} M_{34}} \\
& \sin \left(\omega \tau_{*}\right)=\frac{M_{31} M_{36}-M_{33} M_{34}}{M_{31} M_{35}-M_{32} M_{34}} .
\end{aligned}
$$

Since $\sin ^{2}\left(\omega \tau_{*}\right)+\cos ^{2}\left(\omega \tau_{*}\right)=1$, we have

$$
\omega^{20 q}+k_{19} \omega^{19 q}+\cdots+k_{1} \omega^{q}+k_{0}=0,
$$

where

$$
\begin{aligned}
k_{0}= & \left(a_{0}+d_{0}\right)^{2}\left(a_{0}-d_{0}\right)^{2}-J_{0}^{2}\left(a_{0}-d_{0}\right)^{2}, \\
k_{1}= & 2\left(a_{0}+d_{0}\right)\left(a_{0}-d_{0}\right)\left(\left(a_{0}+d_{0}\right)\left(a_{1}-d_{1}\right) \cos \left(\frac{q \pi}{2}\right)+\left(a_{1}+d_{1}\right)\left(a_{0}-d_{0}\right) \cos \left(\frac{q \pi}{2}\right)\right)- \\
& 2 J_{0}\left(a_{0}-d_{0}\right)\left(J_{1}\left(a_{0}+d_{0}\right) \cos \left(\frac{q \pi}{2}\right)+J_{0}\left(a_{1}-d_{1}\right) \cos \left(\frac{q \pi}{2}\right)\right), \\
k_{19}= & 2\left(a_{4} \cos (2 q \pi) \cos \left(\frac{5 q \pi}{2}\right)\right)-\left(a_{4}-d_{4}\right)\left(\sin (2 q \pi) \sin \left(\frac{5 q \pi}{2}\right)\right) .
\end{aligned}
$$

Let $h_{3}(\omega)=\omega^{20 q}+k_{19} \omega^{19 q}+\cdots+k_{1} \omega^{q}+k_{0}$; obviously, $\lim _{\omega \longrightarrow+\infty} h_{3}(\omega)=+\infty$. When $k_{0}=\left(a_{0}+d_{0}\right)^{2}\left(a_{0}-d_{0}\right)^{2}$ $-J_{0}^{2}\left(a_{0}-d_{0}\right)^{2}<0$, then (42) has at least one positive real root.

Lemma 3. When $\left(a_{0}+d_{0}\right)^{2}\left(a_{0}-d_{0}\right)^{2}-J_{0}^{2}\left(a_{0}-d_{0}\right)^{2}<0$, (42) has at least one positive real root.

By (40), we have

$$
\tau_{*}=\frac{1}{\omega_{30}} \arccos \left(\frac{M_{33} M_{35}-M_{32} M_{36}}{M_{31} M_{35}-M_{32} M_{34}}\right),
$$

where $\pm i \omega_{30}$ is a pair of purely imaginary roots of (37) with $\tau_{* 0}$.

$$
\text { (H3) }\left.\operatorname{Re}\left(\frac{\mathrm{d} \lambda}{\mathrm{d} \tau_{*}}\right)^{-1}\right|_{\tau_{*}=\tau_{* 0}} \neq 0 \text {. }
$$

The derivative of $\lambda$ with respect to $\tau_{*}$ in (37) is easily obtained:

$$
\frac{\mathrm{d} \lambda}{d_{\tau_{*}}}=\frac{\lambda\left(V_{32}+V_{35}\right)}{V_{31}+V_{33}+V_{34}-\tau_{*} V_{13}},
$$

where

$$
\begin{aligned}
V_{31} & =\left(5 q \lambda^{5 q-1}+4 q a_{4} \lambda^{4 q-1}+3 q a_{3} \lambda^{3 q-1}+2 q a_{2} \lambda^{2 q-1}+q a_{1} \lambda^{q-1}\right) e^{\lambda \tau_{*}}, \\
V_{32} & =\left(\lambda^{5 q}+a_{4} \lambda^{4 q}+a_{3} \lambda^{3 q}+a_{2} \lambda^{2 q}+a_{1} \lambda^{q}+a_{0}\right)-e^{\lambda \tau_{*}}, \\
V_{33} & =4 q J_{4} \lambda^{4 q-1}+3 q J_{3} \lambda^{3 q-1}+2 q J_{2} \lambda^{2 q-1}+q J_{1} \lambda^{q-1}, \\
V_{34} & =\left(3 q d_{3} \lambda^{3 q-1}+2 q d_{2} \lambda^{2 q-1}+q d_{1} \lambda^{q-1}\right) e^{-\lambda \tau_{*}}, \\
V_{35} & =\left(d_{3} \lambda^{3 q}+d_{2} \lambda^{2 q}+d_{1} \lambda^{q}+d_{0}\right) e^{-\lambda \tau_{*}}, \\
\left(\frac{d \lambda}{d_{\tau_{*}}}\right)^{-1} & =\frac{V_{31}+V_{33}+V_{34}}{\lambda\left(V_{32}+V_{35}\right)}=-\frac{\tau_{*}}{\lambda}, \\
\left(\frac{d \lambda}{d_{\tau_{*}}}\right)^{-1} & =\frac{W_{30}+W_{31} \cos \left(\omega \tau_{*}\right)+W_{32} \sin \left(\omega \tau_{*}\right)+i\left(W_{33} \cos \left(\omega \tau_{*}\right)+W_{34} \sin \left(\omega \tau_{*}\right)+W_{35}\right)}{W_{36} \cos \left(\omega \tau_{*}\right)+W_{37} \sin \left(\omega \tau_{*}\right)+i\left(-W_{38} \cos \left(\omega \tau_{*}\right)-W_{39} \sin \left(\omega \tau_{*}\right)\right)}-\frac{\tau_{*}}{i W},
\end{aligned}
$$

where 


$$
\begin{aligned}
& W_{30}=4 q J_{4} \omega^{4 q-1} \sin (2 q \pi)+3 q J_{3} \omega^{3 q-1} \sin \left(\frac{3 q \pi}{2}\right)+2 q J_{2} \omega^{2 q-1} \sin (q \pi)+q J_{1} \omega^{q-1} \sin \left(\frac{q \pi}{2}\right), \\
& W_{31}=5 q \omega^{5 q-1} \sin \left(\frac{5 q \pi}{2}\right)+4 q a_{4} \omega^{4 q-1} \sin (2 q \pi)+3 q a_{3} \omega^{3 q-1} \sin \left(\frac{3 q \pi}{2}\right)+2 q a_{2} \omega^{2 q-1} \sin (q \pi)+ \\
& q a_{1} \omega^{q-1} \sin \left(\frac{q \pi}{2}\right)+3 q d_{3} \omega^{3 q-1} \sin \left(\frac{3 q \pi}{2}\right)+2 q d_{2} \omega^{2 q-1} \sin (q \pi)+q d_{1} \omega^{q-1} \sin \left(\frac{q \pi}{2}\right), \\
& W_{32}=5 q \omega^{5 q-1} \cos \left(\frac{5 q \pi}{2}\right)+4 q a_{4} \omega^{4 q-1} \cos (2 q \pi)+3 q a_{3} \omega^{3 q-1} \cos \left(\frac{3 q \pi}{2}\right)+2 q a_{2} \omega^{2 q-1} \cos (q \pi)+ \\
& q a_{1} \omega^{q-1} \cos \left(\frac{q \pi}{2}\right)-\left(3 q d_{3} \omega^{3 q-1} \cos \left(\frac{3 q \pi}{2}\right)+2 q d_{2} \omega^{2 q-1} \cos (q \pi)+q d_{1} \omega^{q-1} \cos \left(\frac{q \pi}{2}\right)\right), \\
& W_{33}=-\left(4 q J_{4} \omega^{4 q-1} \cos (2 q \pi)+3 q J_{3} \omega^{3 q-1} \cos \left(\frac{3 q \pi}{2}\right)+2 q J_{2} \omega^{2 q-1} \cos (q \pi)+q J_{1} \omega^{q-1} \cos \left(\frac{q \pi}{2}\right)\right) \text {, } \\
& W_{34}=-\left(5 q \omega^{5 q-1} \cos \left(\frac{5 q \pi}{2}\right)+4 q a_{4} \omega^{4 q-1} \cos (2 q \pi)+3 q a_{3} \omega^{3 q-1} \cos \left(\frac{3 q \pi}{2}\right)+2 q a_{2} \omega^{2 q-1} \cos (q \pi)+\right. \\
& \left.q a_{1} \omega^{q-1} \cos \left(\frac{q \pi}{2}\right)+3 q d_{3} \omega^{3 q-1} \cos \left(\frac{3 q \pi}{2}\right)+2 q d_{2} \omega^{2 q-1} \cos (q \pi)+q d_{1} \omega^{q-1} \cos \left(\frac{q \pi}{2}\right)\right) \\
& W_{35}=5 q \omega^{5 q-1} \sin \left(\frac{5 q \pi}{2}\right)+4 q a_{4} \omega^{4 q-1} \sin (2 q \pi)+3 q a_{3} \omega^{3 q-1} \sin \left(\frac{3 q \pi}{2}\right)+2 q a_{2} \omega^{2 q-1} \sin (q \pi)+ \\
& q a_{1} \omega^{q-1} \sin \left(\frac{q \pi}{2}\right)-\left(3 q d_{3} \omega^{3 q-1} \sin \left(\frac{3 q \pi}{2}\right)+2 q d_{2} \omega^{2 q-1} \sin (q \pi)+q d_{1} \omega^{q-1} \sin \left(\frac{q \pi}{2}\right)\right), \\
& W_{36}=\omega^{5 q} \sin \left(\frac{5 q \pi}{2}\right)+a_{4} \omega^{4 q} \sin (2 q \pi)+a_{3} \omega^{3 q} \sin \left(\frac{3 q \pi}{2}\right)+a_{2} \omega^{2 q} \sin (q \pi)+a_{1} \omega^{q} \sin \left(\frac{q \pi}{2}\right)- \\
& \left(d_{3} \omega^{3 q} \sin \left(\frac{3 q \pi}{2}\right)+d_{2} \omega^{2 q} \sin (q \pi)+d_{1} \omega^{q} \sin \left(\frac{q \pi}{2}\right)\right) \\
& W_{37}=\omega^{5 q} \cos \left(\frac{5 q \pi}{2}\right)+a_{4} \omega^{4 q} \cos (2 q \pi)+a_{3} \omega^{3 q} \cos \left(\frac{3 q \pi}{2}\right)+a_{2} \omega^{2 q} \cos (q \pi)+a_{1} \omega^{q} \cos \left(\frac{q \pi}{2}\right)+ \\
& a_{0}+d_{3} \omega^{3 q} \cos \left(\frac{3 q \pi}{2}\right)+d_{2} \omega^{2 q} \cos (q \pi)+d_{1} \omega^{q} \cos \left(\frac{q \pi}{2}\right)+d_{0}, \\
& W_{38}=-\left(\omega^{5 q} \cos \left(\frac{5 q \pi}{2}\right)+a_{4} \omega^{4 q} \cos (2 q \pi)+a_{3} \omega^{3 q} \cos \left(\frac{3 q \pi}{2}\right)+a_{2} \omega^{2 q} \cos (q \pi)+a_{1} \omega^{q} \cos \left(\frac{q \pi}{2}\right)+a_{0}\right) \\
& +d_{3} \omega^{3 q} \cos \left(\frac{3 q \pi}{2}\right)+d_{2} \omega^{2 q} \cos (q \pi)+d_{1} \omega^{q} \cos \left(\frac{q \pi}{2}\right)+d_{0} \\
& W_{39}=\omega^{5 q} \sin \left(\frac{5 q \pi}{2}\right)+a_{4} \omega^{4 q} \sin (2 q \pi)+a_{3} \omega^{3 q} \sin \left(\frac{3 q \pi}{2}\right)+a_{2} \omega^{2 q} \sin (q \pi)+ \\
& a_{1} \omega^{q} \sin \left(\frac{q \pi}{2}\right)+d_{3} \omega^{3 q} \sin \left(\frac{3 q \pi}{2}\right)+d_{2} \omega^{2 q} \sin (q \pi)+d_{1} \omega^{q} \sin \left(\frac{q \pi}{2}\right), \\
& R_{e}\left(\frac{d \lambda}{d_{\tau_{*}}}\right)^{-1}=\frac{X_{31} X_{33}+X_{32} X_{34}}{X_{33}^{2}+X_{34}^{2}},
\end{aligned}
$$

where

$$
\begin{aligned}
& X_{31}=W_{30}+W_{31} \cos \left(\omega \tau_{*}\right)+W_{32} \sin \left(\omega \tau_{*}\right), \\
& X_{32}=W_{33}+W_{34} \cos \left(\omega \tau_{*}\right)+W_{35} \sin \left(\omega \tau_{*}\right), \\
& X_{33}=W_{36} \cos \left(\omega \tau_{*}\right)+W_{37} \sin \left(\omega \tau_{*}\right), \\
& X_{34}=W_{38} \cos \left(\omega \tau_{*}\right)-W_{39} \sin \left(\omega \tau_{*}\right) .
\end{aligned}
$$

Noticing that $\operatorname{sign}\left[\left.R_{e}\left(d \lambda / d_{\tau_{*}}\right)\right|_{\tau_{*}=\tau_{* 0}}\right]=\operatorname{sign}[R(d \lambda /$ $\left.\left.d_{\tau_{*}}\right)\left.^{-1}\right|_{\tau_{*}=\tau_{* 0}}\right]$, we can obtain $\left.R_{e}\left(d \lambda / d_{\tau_{*}}\right)\right|_{\tau_{*}=\tau_{* 0}} \neq 0$.
Theorem 3. Suppose that $R_{0}>1$, (16)-(20), (H3), and $\left(a_{0}+d_{0}\right)^{2}\left(a_{0}-d_{0}\right)^{2}-J_{0}^{2}\left(a_{0}-d_{0}\right)^{2}<0$ hold; then, the following results hold:

(i) When $\tau_{*} \in\left[0, \tau_{* 0}\right)$, the endemic equilibrium $\left(S^{*}, E^{*}, I^{*}, K^{*}, R^{*}\right)$ is asymptotically stable.

(ii) When $\tau_{*}=\tau_{* 0}$, system (4) experiences a Hopf bifurcation at the endemic equilibrium. In other words, system (4) has a bifurcation of periodic solution bifurcating near $\tau_{*}=\tau_{* 0}$. 

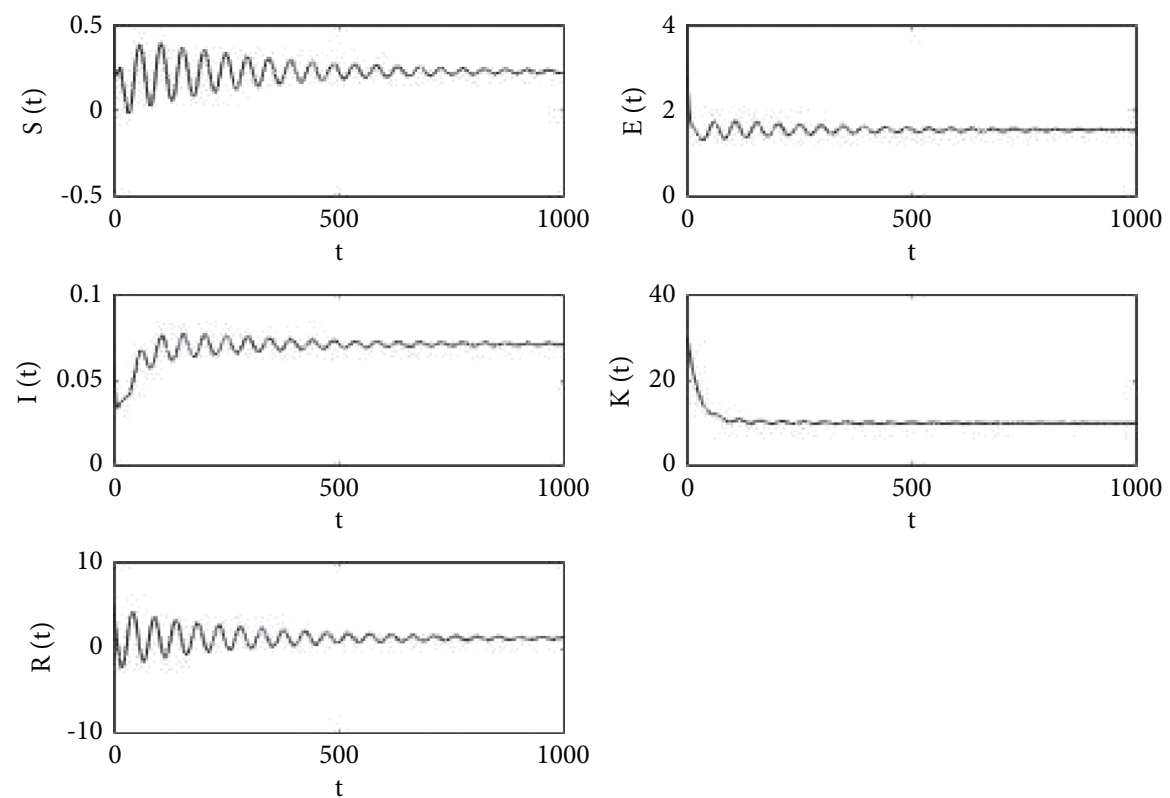

Figure 1: State trajectories of fractional system (4). When $\tau_{1}=15<\tau_{10}$, the equilibrium $(0.5941,2.2161,0.0730,14.3346,2.7823)$ is asymptotically stable.
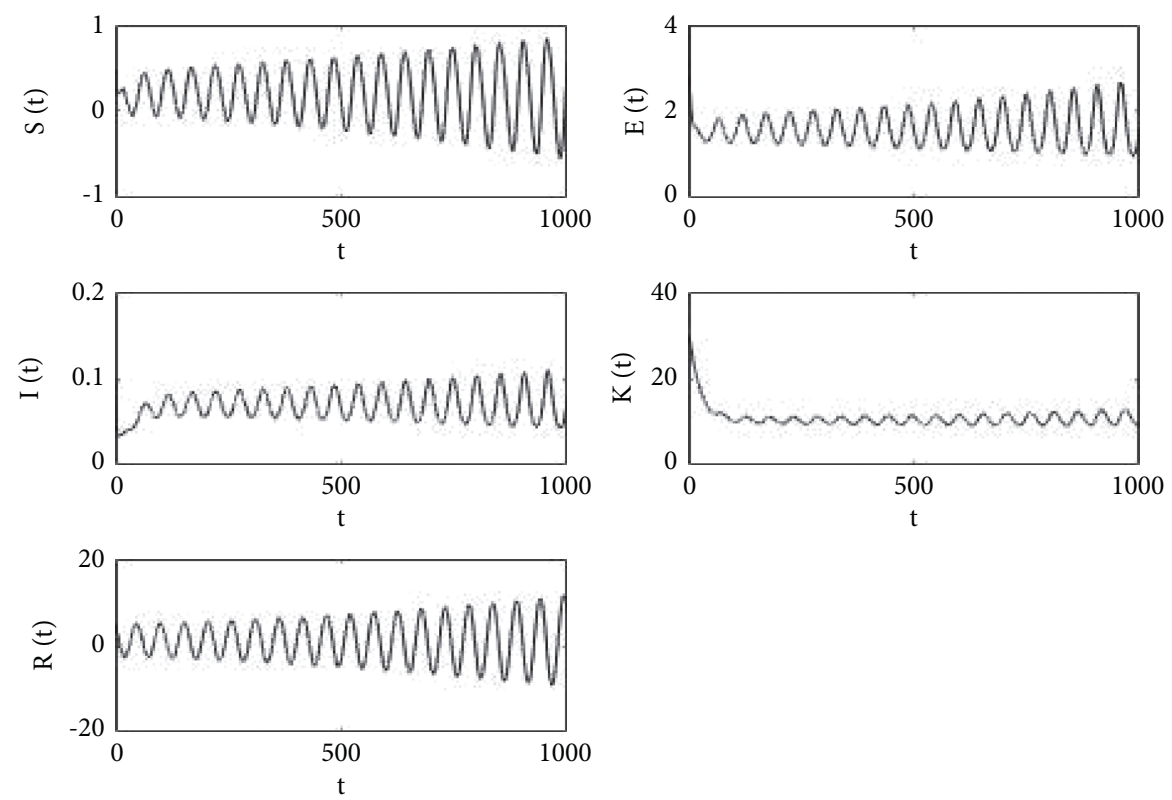

Figure 2: Phase portrait of fractional system (4). When $\tau_{1}=17>\tau_{10}$, the equilibrium $(0.5941,2.2161,0.0730,14.3346,2.7823)$ is not asymptotically stable.

TABLE 1: Relation of parameters of $q, \omega_{10}$, and $\tau_{10}$ of model (4).

\begin{tabular}{lcc}
\hline Fractional order $q$ & Critical frequency $\omega_{10}$ & Bifurcation point $\tau_{10}$ \\
\hline 1 & 0.0874 & 12.9417 \\
0.95 & 0.0779 & 16.1132 \\
0.90 & 0.0711 & 18.4241 \\
0.85 & 0.0641 & 22.0137 \\
0.80 & 0.0570 & 26.9546 \\
0.75 & 0.0499 & 31.3456 \\
0.70 & 0.0427 & 34.5276 \\
\hline
\end{tabular}



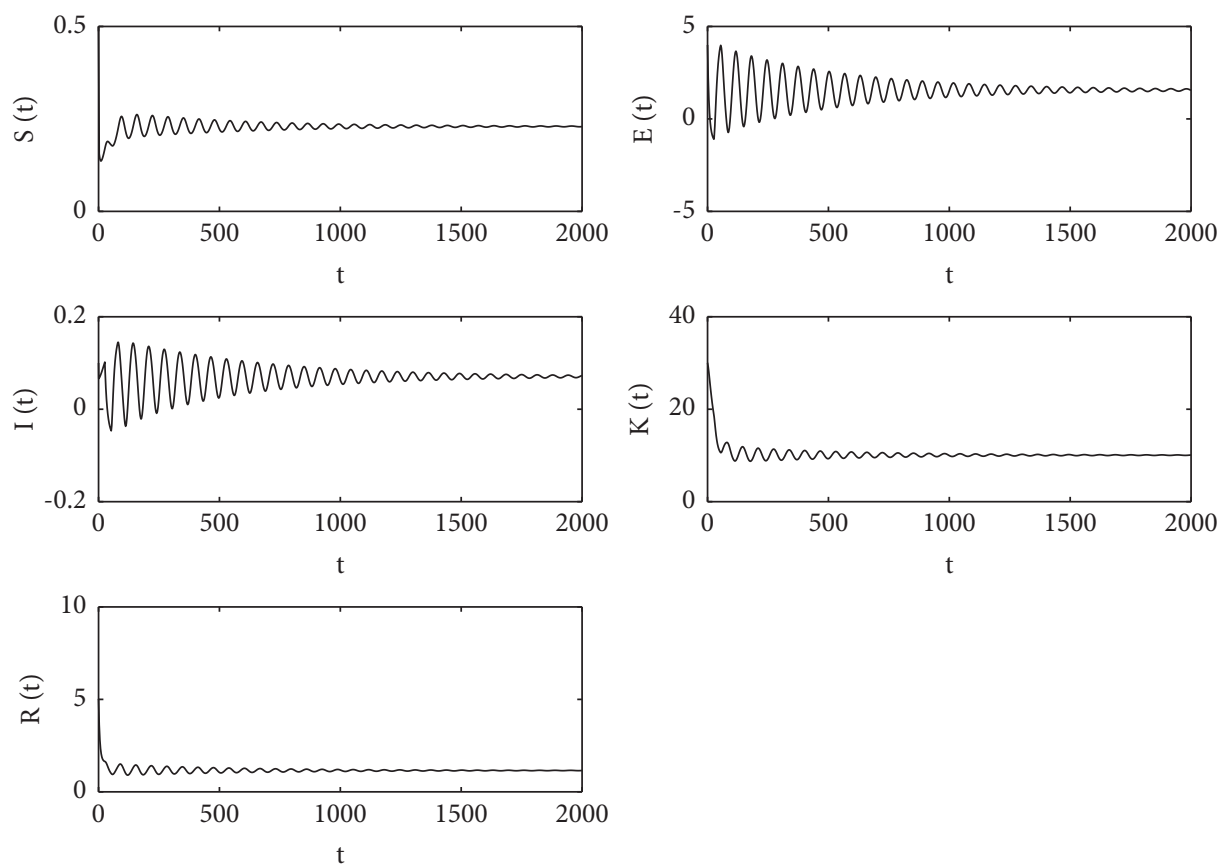

Figure 3: State trajectories of fractional system (4). When $\tau_{2}=26<\tau_{20}$, the equilibrium $(0.5941,2.2161,0.0730,14.3346,2.7823)$ is asymptotically stable.
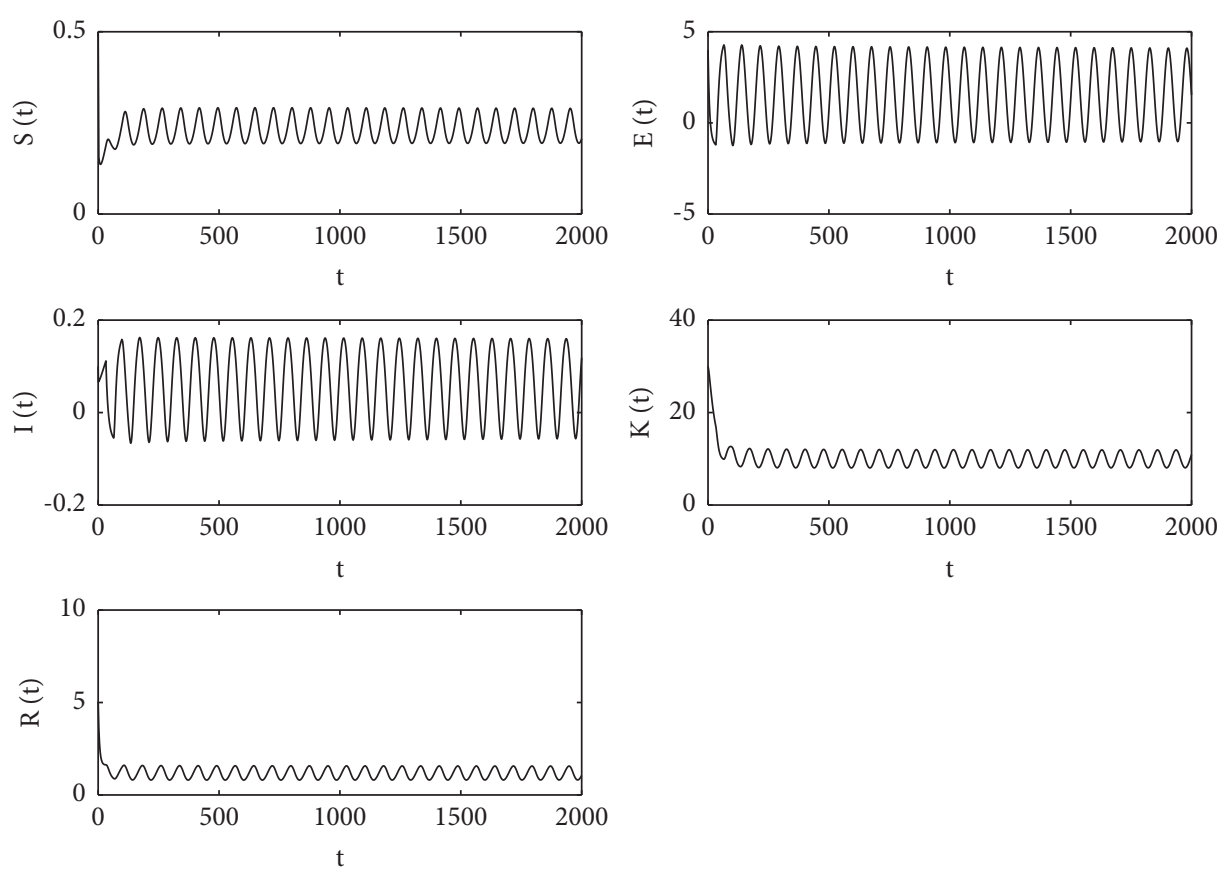

Figure 4: Phase portrait of fractional system (4). When $\tau_{2}=32>\tau_{20}$, the equilibrium $(0.5941,2.2161,0.0730,14.3346,2.7823)$ is not asymptotically stable.

\section{Numerical Simulation and Discussion}

In this part, for confirming our theoretical analysis, we give some numerical results of system (4). Based on time delay fractional-order estimation method [26], we obtain the simulation results with step length $h=0.05$.
In this case, the following case of system (4) with the parameters were considered: $p=0.5, p=0.5, b=1, \beta_{1}=$ $0.25, \quad \beta_{2}=0.3, \quad \phi=0.1, \quad \mu=0.05, \quad \sigma=0.26, \quad \alpha=0.18$, $\chi=0.15, \quad \delta=0.35, \quad \gamma=0.25, \varepsilon=0.15, \quad r=0.5, \quad q=0.95$, and initial values $(0.5,4,0.1,30,5)$ and then we can get a system as follows: 
TABLE 2: Relation of parameters of $q, \omega_{20}$, and $\tau_{20}$ of model (4).

\begin{tabular}{lcc}
\hline Fractional order $q$ & Critical frequency $\omega_{20}$ & Bifurcation point $\tau_{20}$ \\
\hline 1 & 0.0361 & 18.3248 \\
0.95 & 0.0322 & 29.4167 \\
0.90 & 0.0286 & 35.5668 \\
0.85 & 0.0253 & 45.7815 \\
0.80 & 0.0224 & 52.0668 \\
0.75 & 0.0201 & 61.3841 \\
0.70 & 0.0190 & 76.4499 \\
\hline
\end{tabular}
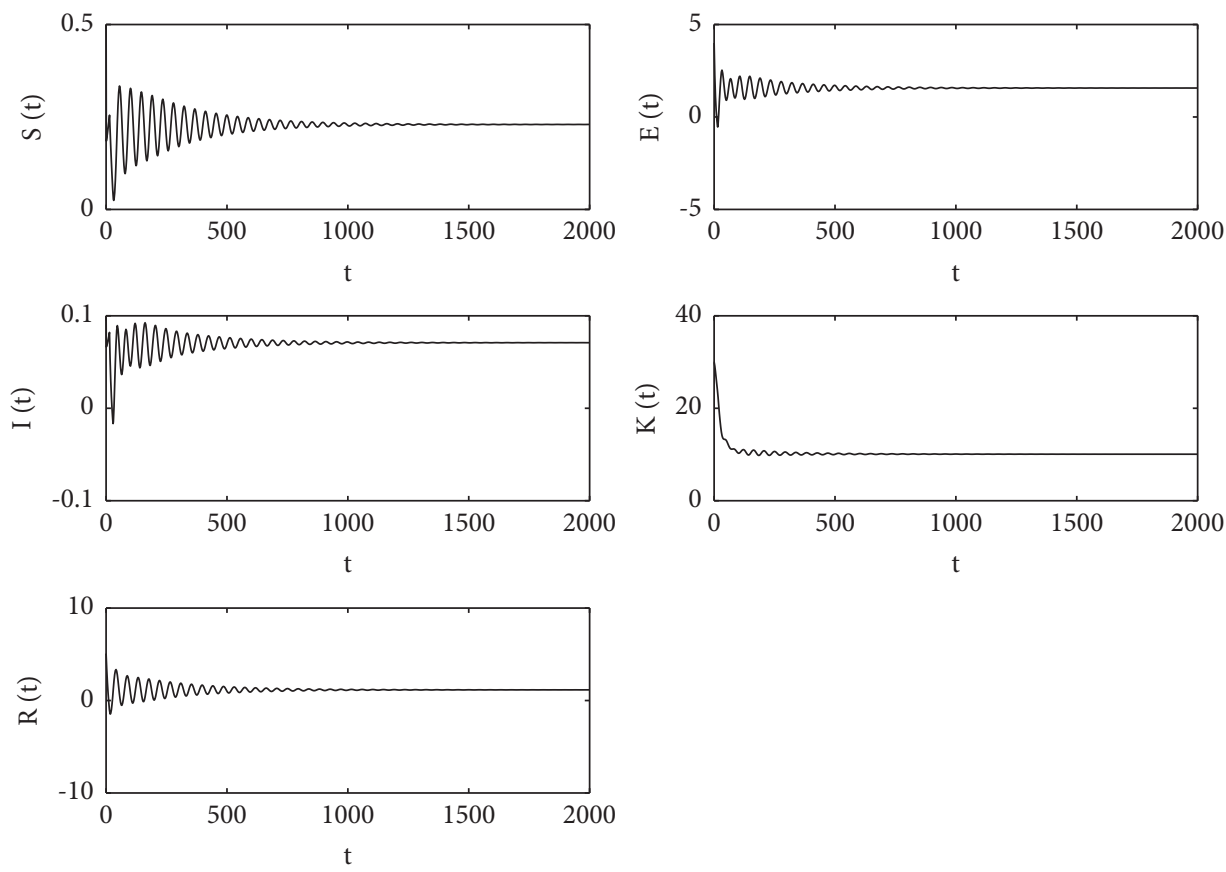

Figure 5: State trajectories of fractional system (4). When $\tau_{*}=14<\tau_{* 0}$, the equilibrium $(0.5941,2.2161,0.0730,14.3346,2.7823)$ is asymptotically stable.
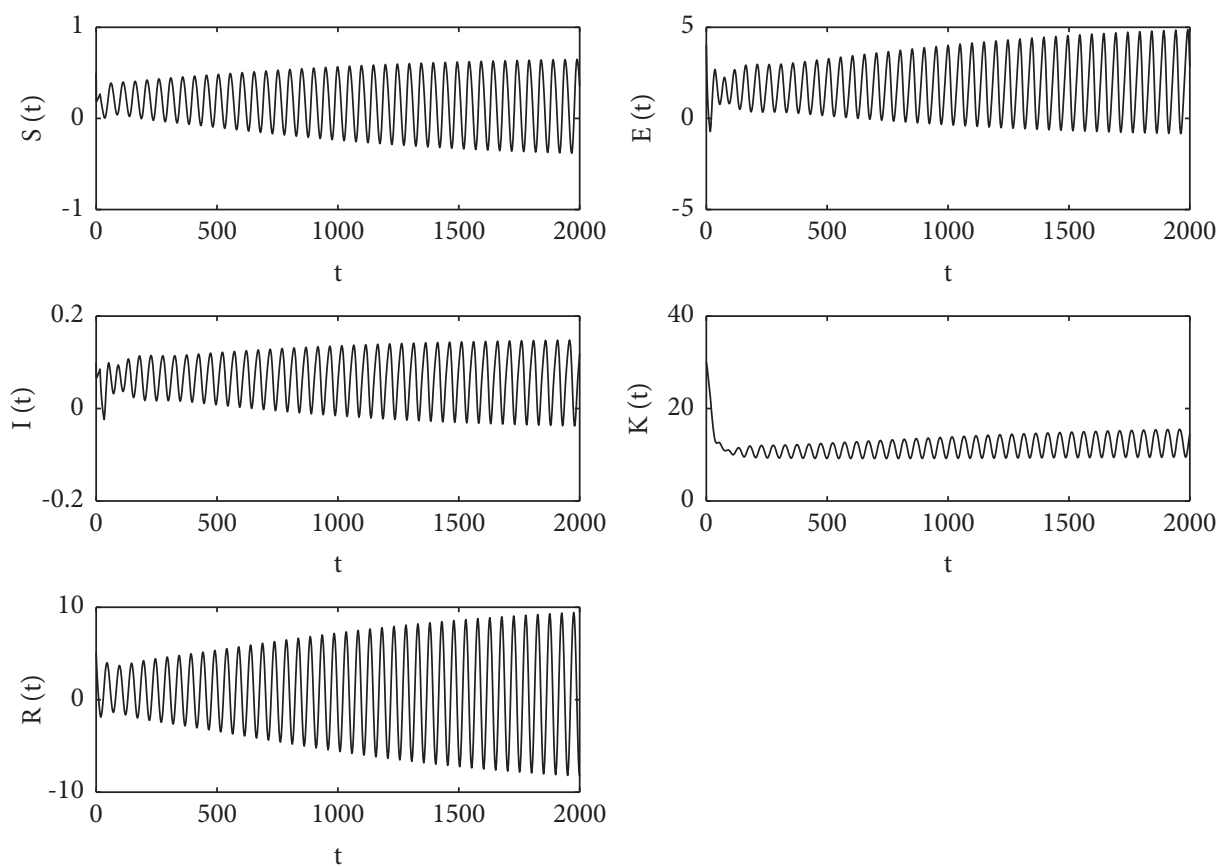

FIgURE 6: Phase portrait of fractional system (4). When $\tau_{*}=16>\tau_{* 0}$, the equilibrium $(0.5941,2.2161,0.0730,14.3346,2.7823)$ is not asymptotically stable. 
TABLE 3: Relation of parameters of $q, \omega_{* 0}$, and $\tau_{* 0}$ of model (4).

\begin{tabular}{lcc}
\hline Fractional order $q$ & Critical frequency $\omega_{* 0}$ & Bifurcation point $\tau_{* 0}$ \\
\hline 1 & 0.0726 & 11.4161 \\
0.95 & 0.0567 & 14.4547 \\
0.90 & 0.0427 & 18.4184 \\
0.85 & 0.0308 & 22.4685 \\
0.8 & 0.0214 & 26.0011 \\
0.75 & 0.0142 & 32.6267 \\
0.7 & 0.0090 & 37.2772 \\
\hline
\end{tabular}

$$
\left\{\begin{array}{l}
D^{0.95} S(t)=0.5-0.25 S(t) E(t)-0.3 S(t) I(t)-0.1 S(t) K(t)-0.05 S(t)+0.26 R\left(t-\tau_{1}\right), \\
D^{0.95} E(t)=0.5+0.25 S(t) E(t)+0.3 S(t) I(t)-0.18 E\left(t-\tau_{2}\right)-0.2 E(t), \\
D^{0.95} I(t)=0.18 E\left(t-\tau_{2}\right)-0.35 I(t) K(t)-0.45 I(t), \\
D^{0.95} K(t)=0.35 I(t) K(t)+0.25 I(t)+0.15 E(t)-0.05 K(t), \\
D^{0.95} R(t)=0.1 S(t) K(t)+0.15 I(t)-0.05 R(t)-0.26 R\left(t-\tau_{1}\right),
\end{array}\right.
$$

which has a unique equilibrium (0.5941, 2.2161, 0.0730, 14.3346, 2.7823).

After calculation, we can obtain $\omega_{10}=0.0779$ and $\tau_{10}=16.1132$. Figure 1 manifests that system (4) is locally asymptotically stable when $\tau_{1} \in\left[0, \tau_{10}\right)$. Figure 2 shows that a Hopf bifurcation exists when $\tau_{1} \in\left[\tau_{10},+\infty\right)$. Table 1 indicates the relation of fractional order $q$ to the critical frequency $\omega_{10}$ and bifurcation point $\tau_{10}$. From Table 1, with decrease in fractional order $q$, the critical frequency $\omega_{10}$ is decreasing and bifurcation point $\tau_{10}$ is increasing.

In the same way, we get $\omega_{20}=0.0322$ and $\tau_{20}=29.4167$. Figure 3 indicates that system (4) is locally asymptotically stable when $\tau_{2} \in\left[0, \tau_{20}\right)$. From Figure 4, system (4) becomes unstable when $\tau_{2} \in\left[\tau_{20},+\infty\right)$. We can see the relation of fractional order $q$ to the critical frequency $\omega_{20}$ and bifurcation point $\tau_{20}$ in Table 2. With decrease in fractional order $q$, the critical frequency $\omega_{20}$ is decreasing and bifurcation point $\tau_{20}$ is increasing, as shown in Table 2 .

To verify the validity of Theorem 3, we take $\omega_{* 0}=0.0567$ and $\tau_{* 0}=14.4547$. From Figures 5 and 6 , we can find that system (4) is locally asymptotically stable when $\tau_{*} \in\left[0, \tau_{* 0}\right)$; but once $\tau_{*}>\tau_{* 0}$, system (4) becomes unstable. We can find the relation of fractional order $q$ to the critical frequency $\omega_{* 0}$ and bifurcation point $\tau_{* 0}$ in Table 3. With decrease in fractional order $q$, the critical frequency $\omega_{* 0}$ is decreasing and bifurcation point $\tau_{* 0}$ is increasing, as shown in Table 3.

From Figures 1-6, we can see the correctness of the theoretical results. All the tables show that the bifurcation point increases gradually with the decrease in fractional order $q$, among which the influence on $\tau_{2}$ is the most obvious. In other words, $\tau_{2}$ is more inclusive than $\tau_{1}$ at the same fractional order $q$. Therefore, when controlling the spread of computer viruses, we should find ways to reduce the temporary immunity time delay in converting recovered computers into susceptible ones.

\section{Conclusions}

We consider a fractional-order model of Susceptible-Exposed-Infected-Kill Signals Recovery (SEIR-KS) computer virus propagation with two delays, based on the model in [25]. The linear method and Laplace transform were used to transform the model. We analyze the coefficient matrix characteristic equation and the system characteristic equation of the linearized system, and then, we get the relationship between the equilibrium stability of the time delay and the fractional-order system; the calculation formula of the critical value of the time delay was derived when Hopf bifurcation appeared. To verify the rationality of the theoretical analysis, the appropriate system parameters are selected for numerical simulation. The simulation results not only verify the correctness of the conclusion but also reveal the influence trend of fractional order on the critical value of time delay. We can find that, with the decrease in fractional order $q$, the bifurcation point increases gradually. The bifurcation point of case 3 is larger than that of case 2 at the same fractional order $q$, which means that we should aim to reduce the temporary immunity time delay for converting recovered computers into susceptible ones. Therefore, the application of this paper is helpful for the computer virus emergency center to make immediate decision and response plan when computing virus outbreak. The research results of this paper extend the research scope and method of the computer virus model to some extent.

\section{Data Availability}

No data were used to support this study.

\section{Conflicts of Interest}

The authors declare that they have no conflicts of interest. 


\section{Acknowledgments}

The authors gratefully acknowledge the financial support provided by the Hunan Natural Science Foundation (2020JJ4516) and the Hunan Provincial Key Foundation of Education Department (17A181).

\section{References}

[1] J. O. Kephart and S. R. White, "Directed-graph epidemiological models of computer viruses," in Proceedings of the IEEE Computer Society Symposium on Research in Security \& Privacy, pp. 343-359, Oakland, CA, USA, May 1991.

[2] B. Guo and S. J. I. Cai, The SIS-BD Model of Computer Virus Spreading on Internet, IEEE, Piscataway, NJ, USA, 2007.

[3] L.-X. Yang and X. Yang, "The effect of infected external computers on the spread of viruses: a compartment modeling study," Physica A: Statistical Mechanics and Its Applications, vol. 392, no. 24, pp. 6523-6535, 2013.

[4] C. Wang Chao, C.-Y. Liu Cheng-Yuan, Y.-P. Hu Yuan-Ping, Z.-H. Liu Zhi-Hong, and J.-F. Ma Jian-Feng, "Stability of information spreading over social network," Acta Physica Sinica, vol. 63, no. 18, Article ID 180501, 2014.

[5] L.-X. Yang, X. Yang, and Y. Y. Tang, "A Bi-virus competing spreading model with generic infection rates," IEEE Transactions on Network Science and Engineering, vol. 5, no. 1, pp. 2-13, 2018.

[6] A. Raza, M. Shoaib Arif, M. Rafiq et al., "Numerical treatment for stochastic computer virus model," Computer Modeling in Engineering \& Sciences, vol. 120, no. 2, pp. 445-465, 2019.

[7] V. MadhuSudanan and R. Geetha, "Dynamics of epidemic computer virus spreading model with delays," Wireless Personal Communications, vol. 115, no. 3, pp. 2047-2061, 2020.

[8] N. Lefevr, A. Kanavos, V. C. Gerogiannis, L. Iliadis, and P. Pintelas, "Employing fuzzy logic to analyze the structure of complex biological and epidemic spreading models," Mathematics, vol. 9, no. 9, p. 977, 2021.

[9] I. Petrá, Fractional-Order Nonlinear Systems: Modeling, Analysis and Simulation, Springer, Berlin, Germany, 2011.

[10] K. Zhang, H. Wang, and H. T. Wang, "Control of a fractionalorder arneodo system," Advanced Materials Research, vol. 383-390, pp. 4405-4412, 2011.

[11] S. D. Lin and C. Lu, "Laplace transform for solving some families of fractional differential equations and its applications," Advances in Difference Equations, vol. 137, 2013.

[12] A. S. Deshpande, V. Daftardar-Gejji, and Y. V. Sukale, "On Hopf bifurcation in fractional dynamical systems," Chaos, Solitons \& Fractals, vol. 98, pp. 189-198, 2017.

[13] X. Teng and Z. Wang, "Stability switches of a class of fractional-delay systems with delay-dependent coefficients," Journal of Computational and Nonlinear Dynamics, vol. 13, no. $11,2018$.

[14] A. Aglić Aljinović, D. Kovačević, D. Kovačević, and M. Puljiz, "Erratum: quantum Montgomery identity and quantum estimates of Ostrowski type inequalities," AIMS Mathematics, vol. 6, no. 1, pp. 675-679, 2021.

[15] C. Xu, Z. Liu, M. Liao, P. Li, Q. Xiao, and S. Yuan, "Fractionalorder bidirectional associate memory (BAM) neural networks with multiple delays: the case of Hopf bifurcation," Mathematics and Computers in Simulation, vol. 182, pp. 471-494, 2021.

[16] C. Xu, M. Liao, P. Li, and S. Yuan, "Impact of leakage delay on bifurcation in fractional-order complex-valued neural networks," Chaos, Solitons \& Fractals, vol. 142, Article ID 110535, 2021.

[17] J. Padisak, "Seasonal succession of phytoplankton in a large shallow lake (balaton, Hungary)--A dynamic approach to ecological memory, its possible role and mechanisms," The Journal of Ecology, vol. 80, no. 2, pp. 217-230, 1992.

[18] V. D. Djordjević, J. Jarić, B. Fabry, J. J. Fredberg, and D. Stamenović, "Fractional derivatives embody essential features of cell rheological behavior," Annals of Biomedical Engineering, vol. 31, no. 6, pp. 692-699, 2003.

[19] H. A. A. El-Saka, "The fractional-order SIS epidemic model with variable population size," Journal of the Egyptian Mathematical Society, vol. 22, no. 1, pp. 50-54, 2014.

[20] E. Uçar, S. Uçar, F. Evirgen, and N. Özdemir, "A fractional SAIDR model in the frame of atangana-baleanu derivative," Fractal and Fractional, vol. 5, no. 2, p. 32, 2021.

[21] M. A. Ansari, D. Arora, and S. P. Ansari, "Chaos control and synchronization of fractional order delay-varying computer virus propagation model," Mathematical Methods in the Applied Sciences, vol. 39, no. 5, pp. 1197-1205, 2016.

[22] M. Xiao, G. Jiang, J. Cao, and W. Zheng, "Local bifurcation analysis of a delayed fractional-order dynamic model of dual congestion control algorithms," IEEE/CAA Journal of Automatica Sinica, vol. 4, no. 2, pp. 361-369, 2017.

[23] W. Deng, C. Li, and J. Lü, "Stability analysis of linear fractional differential system with multiple time delays," Nonlinear Dynamics, vol. 48, no. 4, pp. 409-416, 2006.

[24] R. Gieschke and D. Serafin, "Differential equations in MATLAB," Development of Innovative Drugs via Modeling with MATLAB, Springer, Berlin, Germany, pp. 39-85, 2014.

[25] F. Yang and Z. Zhang, "Hopf bifurcation analysis of SEIR-KS computer virus spreading model with two-delay," Results in Physics, vol. 24, Article ID 104090, 2021.

[26] S. Bhalekar and V. J. g. Daftardar-Gejji, "A predictor-corrector scheme for solving nonlinear delay differential equations of fractional order," Journal of Fractional Calculus and Applications, vol. 1, no. 5, pp. 1-9, 2011. 